BNL-45520

AD/RHIC-95

Conference Paper

\title{
Aperture Determination by Long Term and Multiparticle Tracking
}

\author{
G. F. Dell and G. Parzen
}

May 1991

\section{R H I C P R O J E T}

\author{
Brookhaven National Laboratory \\ Associated Universities, Inc. \\ Upton, NY 11973
}

Under Contract No. DE-AC02-76CH00016 with the UNITED STATES DEPARTMENT OF ENERGY 


\title{
APERTURE DETERMINATION BY LONG TERM \& MULTIPARTICLE TRACKING*
}

\author{
G.F. Dell and G. Parzen \\ Brookhaven National Laboratory \\ Upton, NY 11973, USA
}

\section{Abstract}

Apertures determined by long term tracking for $10^{6}$ turns at $\beta^{*}=2 \mathrm{~m}$ and $6 \mathrm{~m}$ and tracking of 100 particles for 1000 turns at $\beta^{*}=2 \mathrm{~m}$ are reported. The multiparticle results are consistent with the aperture determined from $10^{6}$ turn runs. Finally, the $10^{6}$ turn results are extrapolated to $3 \times 10^{9}$ turns to obtain an estimate of the aperture for which particles survive for 10 hours in RHIC.

\section{INTRODUCTION}

Studies of long term stability in RHIC are reported. The results were obtained from two independent studies using different tracking programs on different computers. Only transverse motion was considered; the effects produced by synchrotron oscillation and tune ripple were not included.

The relation $B=B_{0} \sum(b n+i a n)(X+i Y)^{n}$ was used for the field expansion. Multipoles coefficients $a_{n}$ and $b_{n}$ were generated according to a Gaussian distribution that was truncated at $\pm 3 \sigma$. Apertures, based on the worst of ten cases, are quoted in terms of the initial amplitude $X_{i}$ in a focusing quadrupole having $\beta_{x}=50 \mathrm{~m}$ when the initial emittances in the $X$ and $Y$ planes are equal.

\section{APERTURE DETERMINATION}

\section{A. ORBIT}

Studies with the ORBIT tracking program were made at $\beta^{*}=2 \mathrm{~m}$ and $\beta^{*}=6 \mathrm{~m}$ in all six insertions. Ten distributions of random field errors were used with multipoles of order $2 \leq n \leq 10$ included. Stability at $10^{6}$ turns was determined when an amplitude test of $60 \mathrm{~mm}$ was used as a measure of the dynamic aperture. Runs with one seed were continued until the motion became unstable at $8.8 \times 10^{6}$ turns. The aperture determined by a survival plot is extrapolated to $3 \times 10^{9}$ turns that corresponds to a lifetime of 10 hours in RHIC. The dynamic aperture at $\beta^{*}=2 \mathrm{~m}$ decreases $20 \%$ when runs are extended from 400 to $10^{6}$ turns. A simple extrapolation to $3 \times 10^{9}$ turns, which may be pessimistic, produces an additional $20 \%$ aperture decrease. The results are shown in Figure 1.

Similar runs made with $\beta^{*}=6 \mathrm{~m}$ show little change in the dynamic aperture as runs are extended from 400 to $10^{6}$ turns. Survival plots for $\beta^{*}=6 \mathrm{~m}$ are shown in Figure 2.

\footnotetext{
- Work performed under the auspcies of the U.S. Department of Energy.
}

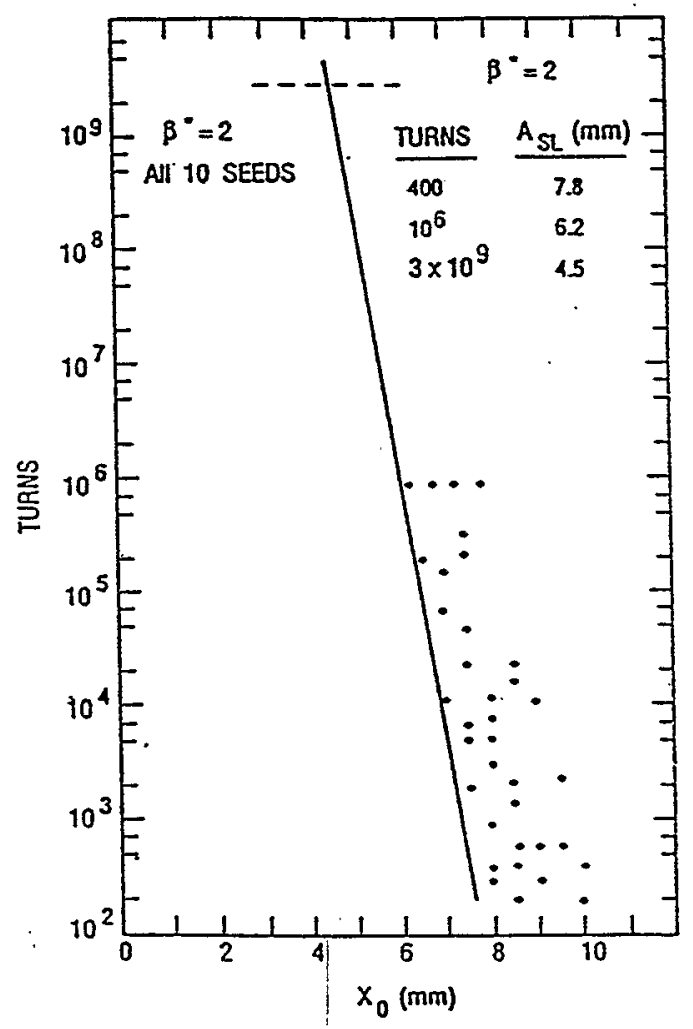

Figure 1: Survival times versus particle amplitude $X_{0}$. $\beta^{*}=2 \mathrm{~m}$. Ten distributions of random field errors.

\section{B. PATRICIA}

Aperture studies were made with the PATRICIA program with $\beta^{*}=2 \mathrm{~m}$ in all insertions and the tune was $\nu_{x}=28.826$ and $\nu_{y}=28.820$. Multipole expansions for fields were made for $2 \leq n \leq 16$ in dipoles and $2 \leq n \leq 10$ in quadrupoles; multipoles were present in all elements of the arcs and insertions. The amplitude was tested at every element to assure the particle remains within the vacuum chamber.

\section{B.1. Long Term Tracking}

Stability at $10^{6}$ turns was determined for ten distributions of random field errors when test particles were launched with $\epsilon_{x}=\epsilon_{y}$ and $X^{\prime}=Y^{\prime}=0$. Histories for the worst cases, seeds \#9 and \#10, and the best case, seed 
\#1, are shown in Figure 3. Points at values $<10^{6}$ indicate failure; points at $10^{6}$ turns indicate survival. Some seeds indicate sharp reductions in survival as the initial amplitude is decreased. For seed \# 9 the particle survived for $84 \mathrm{~K}$ turns at $X_{i}=7.3 \mathrm{~mm}$ but failed after 6,130 turns at an amplitude of $6.7 \mathrm{~mm}$. For $10^{6}$ turn runs, the acceptance of RHIC is $6.2 \mathrm{~mm}$ as compared with $7.6 \mathrm{~mm}$ for 1000 turn runs.

\section{B.2. Multiparticle Tracking}

Determination of $\epsilon_{x}$ and $\epsilon_{y}$ after each turn indicates there is transfer of emittance between the $X$ and $Y$ planes and that the total emittance is not always conserved. This is shown in Figure 4. The emittance transfer is repetitive with a period of $\cong 150$ turns but does not include points having- $\epsilon_{x} / \epsilon_{t}(o) \cong 1$. Runs were made to determine whether the aperture is independent of the initial coordinates $\left(X, X^{\prime}, Y, Y^{\prime}\right)$ of the particles. We select $\epsilon_{x}$ randomly in the range $0<\epsilon_{z}<\epsilon_{t}(o)$, use $\epsilon_{y}=\epsilon_{t}(o)-\epsilon_{x}$, and determine $X, X^{\prime}$, consistent with $\epsilon_{x}$, and $Y, Y^{\prime}$, consistent with $\epsilon_{y}$.

At each amplitude, 100 particles were tracked for 1000 turns. Tracking was performed at the total emittance $\epsilon_{t}(o)$ for which particles, launched with $\epsilon_{x}=\epsilon_{y}$ and $X^{\prime}=Y^{\prime}=0$, survived $10^{6}$ turns. Of the ten cases studied, five were stable, two showed $2.5 \%$ aperture decrease, and three showed aperture decreases between 8 and 14\%. For

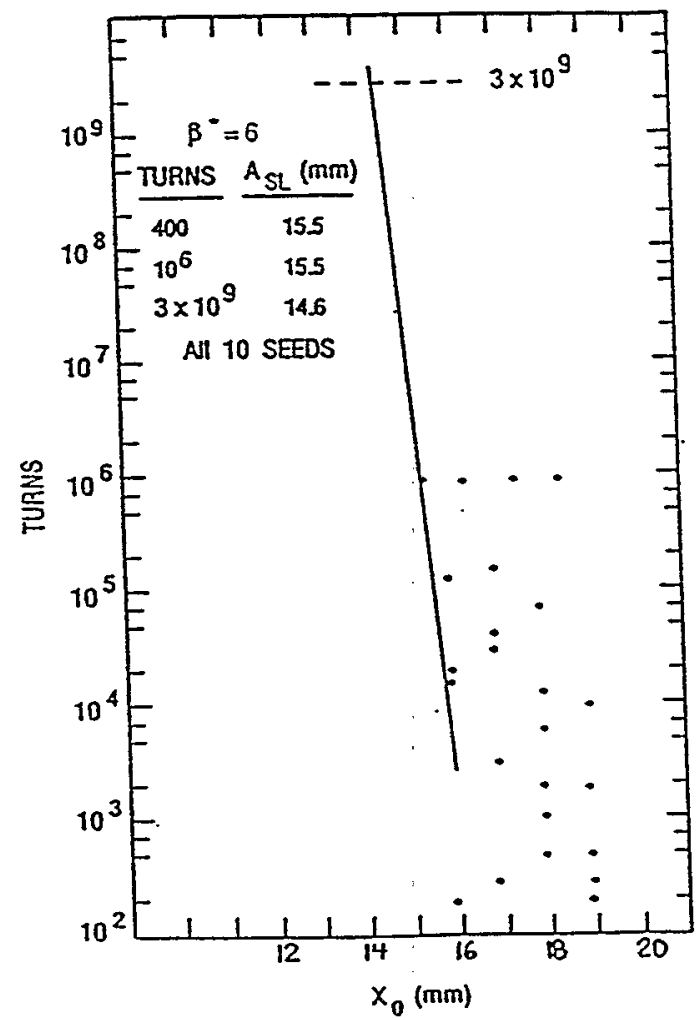

Figure 2: Survival times versus particle amplitude $X_{o} . \beta^{*}=6 \mathrm{~m}$. Ten distributions of random field errors.
RN\#1, the machine showing the largest aperture for the $10^{6}$ turn runs, 35 of the 100 particles failed within 1000 turns.

The sequence of random numbers used for multiparticle launching differs for different seeds, and hence particles are launched with different $X, X^{\prime}, Y$ and $Y^{\prime}$. This possible source of difference was checked by resetting the random number generator of all machines to the value used for the machine having the smallest aperture. This gives the same relative $X, X^{\prime}, Y$ and $Y^{\prime}$ for each machine. The five machines showing stability again had no failures when 100 particles were tracked for 1000 turns.

A test was also made to determine if the aperture for these five machines is larger than that obtained from long term tracking. This was confirmed; a comparison of results from $10^{6}$ turn runs and multiparticle runs are shown in Figure 5.

\section{DISCUSSION}

For the $10^{6}$ runs, particles were launched with $\epsilon_{x}=$ $\epsilon_{y}=\epsilon_{t} / 2$. These runs most often showed more complete emittance transfer to the $Y$ plane than to the $X$ plane. For multiparticle tracking, no limitation was placed on the initial ratio of $\epsilon_{x} / \epsilon_{t}$. Some failures result from particles with $\epsilon_{x} / \epsilon_{t}$ outside the range observed during the $1 \times 10^{6}$ turn studies. Four out of five seeds showing decreased

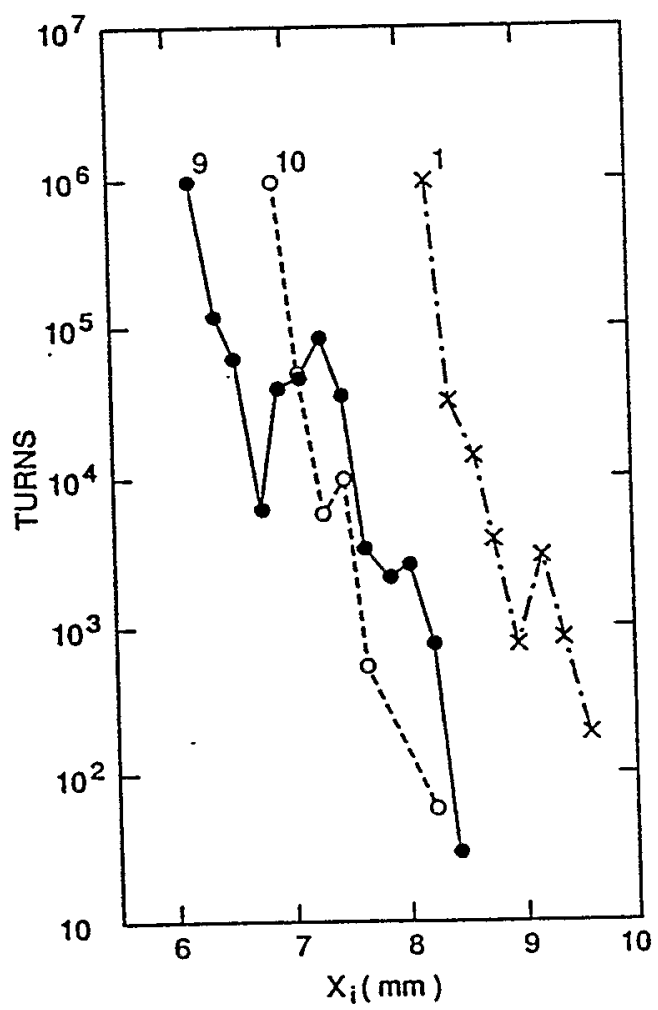

Figure 3: Dependence of particle survival on initial amplitude for three distributions of random field errors: worst cases (9\&10), and best case (1). 


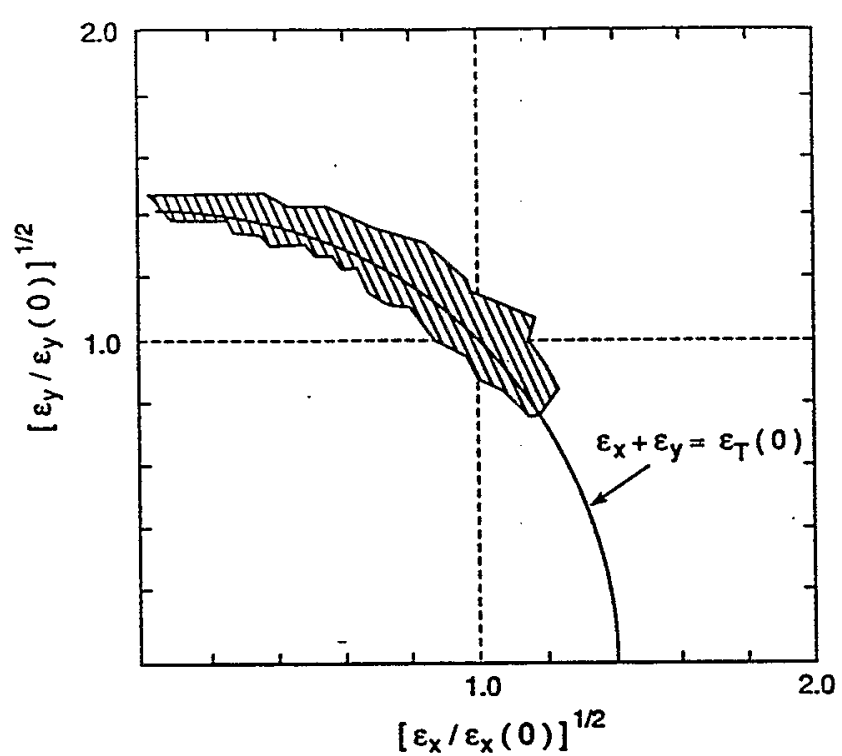

Figure 4: Dependence of $\sqrt{\epsilon_{y}}$ on $\sqrt{\epsilon_{x}}$ during a 500 turn run. Seed \#5.

Table 1: Summary of Aperture Determinations.

\begin{tabular}{|ccccc|}
\hline & $\beta^{*}(\mathrm{~m})$ & $\begin{array}{c}400 \\
(\mathrm{~mm})\end{array}$ & $\begin{array}{c}10^{6} \\
(\mathrm{~mm})\end{array}$ & $\begin{array}{c}1000(\mathrm{MP}) \\
(\mathrm{mm})\end{array}$ \\
\hline ORBIT & 2 & 7.8 & 6.2 & \\
& 2 & 7.6 & 6.2 & 6.2 \\
PATRICIA & 2 & & & \\
ORBIT & 6 & 15.5 & 15.5 & \\
\hline
\end{tabular}

aperture for the multiparticle runs were launched with $\epsilon_{t} / \epsilon_{t} \geq 0.84$ (a region infrequently encountered in the $10^{6}$ turn runs), and four of five seeds showing increased aperture had $\epsilon_{x} / \epsilon_{t} \leq \mathbf{0 . 5 3}$ a region frequently encountered in the $10^{6}$ turn runs.

We conclude that multiparticle tracking using many sets of launching coordinates can provide a test of particle stability in regions where launching with $\epsilon_{x}=\epsilon_{y}$ and $X^{\prime}=$ $Y^{\prime}=0$ seems stable. An example is provided by machine \#5 for which failure at $X=6.9 \mathrm{~mm}$ was experienced after

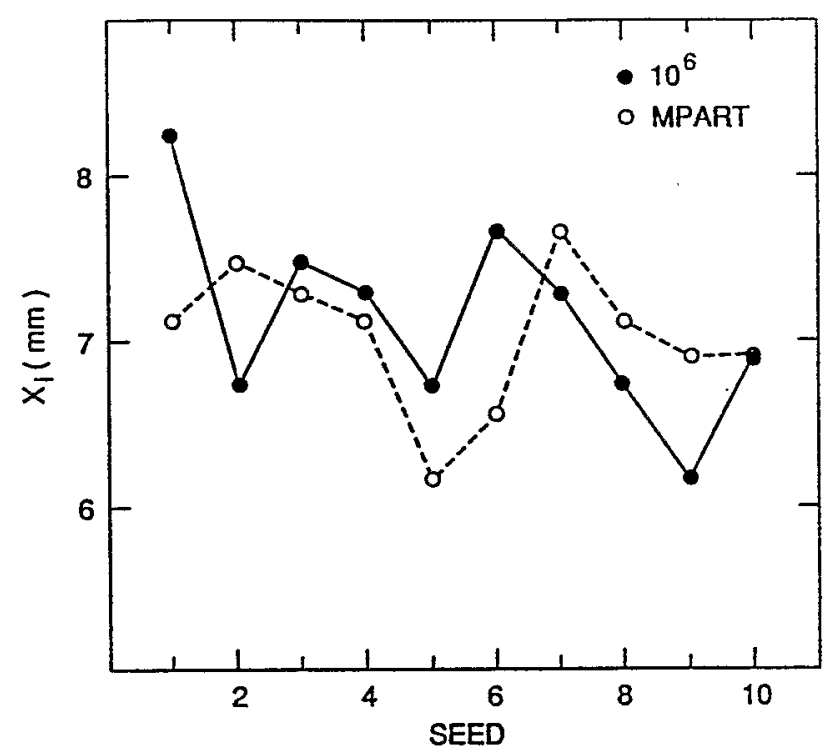

Figure 5: Comparison of aperture determinations from long term $\left(10^{6}\right)$ and multiparticle (MP) tracking runs for ten distributions of random field errors.

$573 \mathrm{~K}$ turns, while 17 of 100 particles having the same emittance failed within 1000 turns when launched in the multiparticle mode. Multiparticle tracking complements long term tracking by probing areas of $\left(X, X^{\prime}, Y, Y^{\prime}\right)$ space not available to particles launched with $\epsilon_{x}=\epsilon_{y}$ and $X^{\prime}=Y^{\prime}=0$. The results are summarized in Table 1 .

\section{ACKNOWLEDGMENTS}

The authors wish to acknowledge the help provided from reading the reports on tracking studies by $T$. Garavaglia, S.K. Kauffmann, R. Stiening, and D.M. Ritson, ${ }^{1}$ and V. Visnjic. ${ }^{2}$

\section{REFERENCES}

[1] T. Garavaglia, S.K. Kauffmann, and R. Stiening, SSC Report SSCL-269 (1990).

T. Garavaglia, S.K. Kauffmann, R. Stiening, and D.M. Ritson, SSC Report SSCL-268 (1990).

[2] V. Visnjic, Fermilab Publication 90/80 (1990). 